\title{
Entropy
}

ISSN 1099-4300

www.mdpi.org/entropy/

\section{On the Entropy Production Due to Explosion in Seawater}

\author{
R.P.Yadav ${ }^{1}$, P.K.Agarwal ${ }^{2}$ and Atul Sharma ${ }^{3, *}$ \\ 1. Department of Physics, Govt. P.G. College, Bisalpur (Pilibhit), U.P., India \\ 2. Department of Physics, D.A.V. College, Muzaffarnagar, U.P., India \\ 3. Department of Physics, V.S.P. Govt. College, Kairana (Muzaffarnagar), U.P., India \\ * Email addresses of authors: \\ rpyadav93PPhysics@yahoo.com (R.P.Yadav), pkaphysics@yahoo.com (P.K.Agarwal)and \\ attulsharma@yahoo.co.in (Atul Sharma)
}

Received: 7 March 2005 / Accepted: 10 May 2005 / Published: 12 May 2005

\begin{abstract}
The change in entropy is calculated due to propagation of blast waves, produced by the explosion of spherical charge in sea water, using the energy hypothesis of Thomas. The release of energy is considered as instantaneous and the gravitation of earth is taken into account, assuming the earth to be a sphere of uniform density. For the sake of simplicity, effect of rotation of the earth is not considered. The explosion is considered at different depths. It has been found that the change in entropy of water decreases at different radial points, as the shock moves away from the point of explosion. Explosion occurred at larger depths, produces a smaller change in entropy of water, then the explosion of same energy, at smaller depths. Directional dependence of entropy production and the motion of the shock are also studied. It has been found that, entropy production is larger in upward motion of the underwater shock. However the shock velocity increases in downward direction.
\end{abstract}

Keywords: explosion, entropy, underwater shock, Tsunami waves

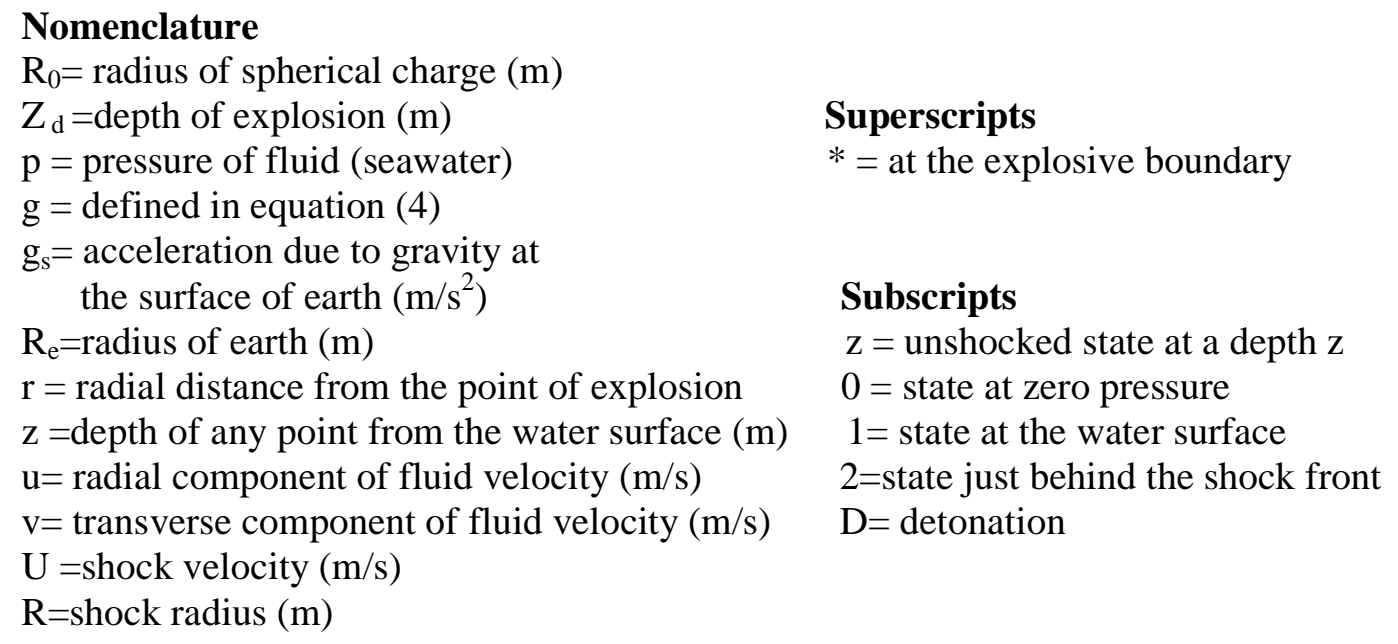


$\mathrm{R}^{\prime}=$ nondimensional shock radius $\left(=\mathrm{R} / \mathrm{R}_{0}\right)$

$\mathrm{n}=\mathrm{a}$ constant for water

$\mathrm{B}(s)=$ slowly varying function of entropy, normally considered as constant $(\mathrm{kb})$

$\mathrm{T}^{\prime}=$ energy released during explosion $(\mathrm{J})$

$\mathrm{U}_{\mathrm{D}}=$ detonation velocity $(\mathrm{m} / \mathrm{s})$

$c_{p}=$ specific heat of water $(\mathrm{kJ} / \mathrm{kg}-\mathrm{K})$

$\mathrm{T}=$ absolute temperature $(\mathrm{K})$

$\mathrm{E}=$ internal energy/unit mass $(\mathrm{J} / \mathrm{kg})$

$\mathrm{V}=$ specific volume $\left(\mathrm{m}^{3} / \mathrm{kg}\right)$

$\mathrm{W}=$ work, defined in equation (29)

$s=$ specific entropy (entropy /unit mass)(kJ/kg-K)

$\Delta \mathrm{Q}=$ heat flow / unit mass $(\mathrm{J} / \mathrm{kg})$

\section{Introduction}

During and after Second World War, many scientists have shown their interest in analyzing theoretically and experimentally the explosions in different types of media. Recently, Tsunami waves had produced a disastrous effect on the human lives, in the coastal areas of south east Asia, which are caused by shock waves due to submarine earthquake. Underwater explosions and shock waves have been studied earlier mainly by Kirkwood and Bethe [12], Penny and Dasgupta [14], Kirwood and Brinkley [13]. Later, Hunter [11], Berger and Holt [1], Bhatnagar et al. [2], Ranga Rav et al. [16], Singh et al. [21,22], Singh [18,19,20] and Vishvakarma et al. [25] have studied shock propagation in the water. Many methods are developed for the study of shock waves. Thomas [24] used 'Energy hypothesis' for spherical blast waves. This hypothesis was successfully applied by Bhutani [3] to cylindrical blast waves in Magnetogasdynamics. A theoretical study of explosion in water (without considering the effect of gravity) is carried out by Singh et al. [21] and Singh [18] using Energy hypothesis. Experimental verification of Energy hypothesis is given by Singh et al. [22] and Singh [20]. Vishvakarma et al. [25] used Energy hypothesis to explosion problem in sea water considering the effect of gravity.

In most of the underwater shock research stated above, entropy change at the shock front is considered negligible. This approximation of negligible entropy change during shock propagation in water is also supported by the fact that the equations for conservation of mass and momentum, across a shock (the mechanical conditions) together with the equation of state for the fluid are sufficient to determine the shock process. However a definite amount of mechanical energy of the shock wave is converted into heat, producing entropy. In case of strong shocks, it cannot be neglected [11]. To calculate it, equation for conservation of energy is also needed [7].

In the present paper, an attempt has been made to study the change in entropy of seawater due to the propagation of blast waves generated by strong explosion. The energy released during explosion is considered instantaneous and its distribution is uniform in the shocked field. It is also assumed that the whole energy liberated during explosion is used in the propagation of the shock. The basic equations of Vishvakarma et al. [25] are considered, in which Energy hypothesis deviced by Thomas [24] is used. This hypothesis is well suited to express, at least to a first approximation, the basic property of energy behavior, within the blast caused by the explosion. 


\section{Basic formulation}

Let the explosion take place due to a spherical charge, at a time $t=0$ and at a depth ' $\mathrm{Z}_{d}$ ' from the surface of water. This point is taken as origin. This explosion produces spherical blast waves (fig.1).

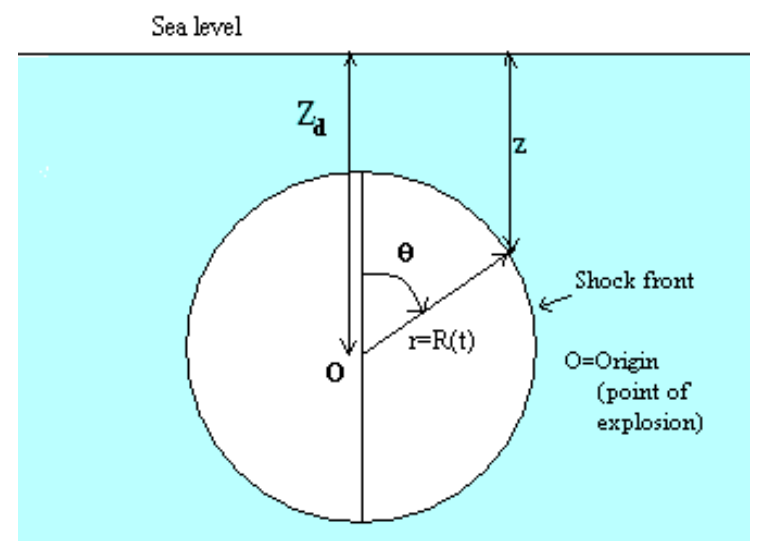

Figure 1 - Shock front in the seawater

The partial differential equations representing the conservation of mass and momentum of the flow are given by:

$$
\begin{aligned}
& \frac{\partial \rho}{\partial t}+\frac{\partial}{\partial r}(\rho u)+\frac{1}{r} \frac{\partial}{\partial \theta}(\rho v)+\frac{2 \rho u}{r}+\frac{\cot \theta}{r}(\rho v)=0 \\
& \frac{\partial u}{\partial t}+u \frac{\partial u}{\partial r}+\frac{v}{r} \frac{\partial u}{\partial \theta}+\frac{1}{\rho} \frac{\partial p}{\partial r}+g \cos \theta=0 \\
& \frac{\partial v}{\partial t}+u \frac{\partial v}{\partial r}+\frac{v}{r} \frac{\partial v}{\partial \theta}+\frac{1}{\rho r} \frac{\partial p}{\partial \theta}-g \sin \theta=0
\end{aligned}
$$

Assuming the earth to be a homogeneous sphere (uniform density), ' $g$ ' varies only with the depth from the surface of the earth, not due to non uniform density [17].The variation of acceleration due to gravity ' $g$ ' at a depth ' $z$ ' below the earth surface is given by:

$$
g=g_{s}\left(1-\frac{z}{R_{e}}\right)
$$

In the stationary conditions $u=0$ and $v=0$ we have:

$$
\begin{aligned}
& \frac{1}{\rho} \frac{\partial p}{\partial r}=-g \cos \theta \\
& \frac{1}{\rho r} \frac{\partial p}{\partial \theta}=g \sin \theta
\end{aligned}
$$

The depth ' $z$ ' at any point on the shock front $[r=R(t)]$ is (figure 1 ):

$$
z=z_{d}-R \cos \theta
$$

Where ' $\theta$ ' is the angle between vertical direction and the shock radius ( $\mathrm{R}$ ) corresponding to the point. 
Equation of state for sea water is:

$$
p=B(s)\left[\left(\frac{\rho}{\rho_{0}}\right)^{n}-1\right]
$$

From $(8), p_{z}$ is given by:

$$
p_{z}=B(s)\left[\left(\frac{\rho_{z}}{\rho_{0}}\right)^{n}-1\right]
$$

From (8) and (9) we have:

$$
p-p_{z}=\left(B+p_{z}\right)\left[\left(\frac{\rho}{\rho_{z}}\right)^{n}-1\right]
$$

Similarly, with pressure and density at water surface, we get:

$$
p-p_{1}=\left(B+p_{1}\right)\left[\left(\frac{\rho}{\rho_{1}}\right)^{n}-1\right]
$$

Solving equation (4), (5), (7) and (11), we get the values of $\rho_{z}$ and $p_{z}$ as:

$$
\begin{aligned}
& \rho_{z}=\rho_{1}\left[1+K\left(R_{e}-z\right) z\right] \frac{1}{(n-1)} \\
& p_{z}=\left(B+p_{1}\right)\left[\left\{1+K\left(R_{e}-z\right) z\right\} \frac{n}{(n-1)}-1\right]+p_{1} \\
& \text { where } K=\frac{(n-1)}{n} \frac{\rho_{1}}{\left(B+p_{1}\right)} \frac{g_{s}}{R_{e}}
\end{aligned}
$$

As the shock travels forward, the spherical front changes its shape [6]. It is assumed that the deviation of shock front from its spherical shape is negligible. Hence the transverse component $v$ of particle velocity, at the shock front is always tangent to it and it is unchanged during shock transition.

Taking unshocked water as stationary, the Rankine -Hugoniot conditions relating the fluid parameters, in front and behind the shock front are given by:

$$
\begin{aligned}
& \rho_{2}\left(U-u_{2}\right)=\rho_{z} U \\
& p_{2}-p_{z}=\rho_{z} U u_{2} \\
& E_{2}-E_{z}=\frac{p_{2} u_{2}}{\rho_{z} U}-\frac{1}{2} u_{2}^{2}
\end{aligned}
$$

With compression ratio $\delta=\left(\rho_{2} / \rho_{z}\right)$, we have the jump conditions as follows:

$$
\begin{aligned}
& p_{2}=\left(B+p_{z}\right)\left[\delta^{n}-1\right]+p_{z} \\
& U=\left[\frac{\left(B+p_{z}\right)\left(\delta^{n}-1\right) \delta}{\rho_{z}(\delta-1)}\right]^{\frac{1}{2}}
\end{aligned}
$$




$$
u_{2}=\left[\frac{\left(B+p_{z}\right)\left(\delta^{n}-1\right)(\delta-1)}{\rho_{z} \delta}\right]^{\frac{1}{2}}
$$

These are four relations [(from (17) to (20)] in five unknown $p_{2}, u_{2}, U, \rho_{2}$ and $E_{2}$. To solve these, another fifth relation is needed.

Energy hypothesis [24] provides this fifth relation. According to it, change in total energy per unit mass of water at a distance $R$ from the centre of explosion due to the propagation of spherical shock is given by:

$$
\varepsilon_{2}-\varepsilon_{z}=\frac{3 \alpha T^{\prime}}{4 \pi \rho_{2} R^{3}}
$$

$\alpha$ is a constant given by [18]:

$$
\alpha=\lim _{R \rightarrow R_{0}} \frac{4 \pi R^{3} \rho_{2}}{3 T^{\prime}}\left(\varepsilon_{2}-\varepsilon_{z}\right)
$$

From equation (17) and (21) we get:

$$
\frac{p_{2} u_{2}}{\rho_{z} U}=\frac{3 \alpha T^{\prime}}{4 \pi \rho_{2} R^{3}}
$$

Putting the values of $p_{2}, u_{2}$ and $U$ from (18), (19) and (20) in (23) we get:

$$
\left\{\left(B+p_{z}\right)\left(\delta^{n}-1\right)+p_{z}\right\}(\delta-1)=\frac{3 \alpha T^{\prime}}{4 \pi R^{3}}
$$

At the boundary of spherical charge (at $R=R_{0}$ ), we have $\delta=\delta^{*}$, then:

$$
\left\{\left(B+p_{z}^{*}\right)\left(\delta^{* n}-1\right)+p_{z}^{*}\right\}\left(\delta^{*}-1\right)=\frac{3 \alpha T^{\prime}}{4 \pi R_{0}^{3}}
$$

Dividing (24) by (25) we get:

$$
\frac{\left\{\left(B+p_{z}\right)\left(\delta^{n}-1\right)+p_{z}\right\}(\delta-1)}{\left\{\left(B+p_{z}^{*}\right)\left(\delta^{* n}-1\right)+p_{z}^{*}\right\}\left(\delta^{*}-1\right)}=\frac{1}{R^{\prime 3}}
$$

$\delta^{*}$ is calculated using the mismatch method due to Butchanan and James [5].According to it, at the explosive boundary:

$$
\frac{p_{2}^{*}}{p_{D}}=\frac{2 \rho_{z} U^{*}}{\rho_{D} U_{D}+\rho_{z} U^{*}}
$$

The values of $\rho_{D}, p_{D}$ and $U_{D}$ are available from detonation data for a known quantity of a particular explosive. Then for a particular direction $\theta$, the fluid parameters $\delta, p_{2}, u_{2}$, and $U$ are calculated at different $R^{\prime}$ values, using equations (26), (18), (19) and (20) [9]. 
Now according to the first law of thermodynamics, heat flow during an irreversible process is [26]:

$$
\Delta Q=\left(E_{2}-E_{z}\right)-W
$$

Work done $W$, by the shock wave on the unit mass of seawater in compressing it is given by:

$$
W=-\int_{z}^{2} p d V
$$

Using equation of state (8), (29) and (28), we get:

$$
\Delta Q=\Delta E-\left[\frac{B}{\rho_{z}}\left(\frac{1}{\delta}-1\right)+\frac{\left(B+p_{z}\right)}{(n-1) \rho_{z}}\left\{\delta^{(n-1)}-1\right\}\right]
$$

The well known thermodynamic relation is:

$$
d Q=c_{p} d T-T\left(\frac{\partial V}{\partial T}\right)_{p} d p
$$

At the shock front, both temperature and pressure are changing. However in case of solid and liquids, an increase of pressure produces only a small temperature change. Also experiments show that $\mathrm{c}_{p}$ hardly changes even for an increase 10,000 Bars [26]. So for unit mass of seawater, the heat flow can be approximated as:

$$
d Q \cong c_{p} d T
$$

From Second law of Thermodynamics:

$$
d Q \cong T d s
$$

From (32) and (33), for the states above and behind the shock front, we get:

$$
\Delta s=\int_{z}^{2} d s=c_{p} \int_{T_{z}}^{T_{2}} \frac{d T}{T}=c_{p} \ln \left(\frac{T_{2}}{T_{z}}\right)
$$

.Also from (32), we have:

$$
\Delta Q=c_{p}\left(T_{2}-T_{z}\right)
$$

From (34), (35) and (30) we get finally the 'rate of specific entropy production' $\{$ It must be remembered that R-H conditions (15), (16) and (17) are defined for unit area of shock wave per unit time across the shock front is given as:

$$
\Delta s=c_{p} \ln \left[1+\frac{\Delta E}{c_{p} T_{z}}-\frac{1}{c_{p} T_{z}}\left\{\frac{B}{\rho_{z}}\left(\frac{1}{\delta}-1\right)+\frac{\left(B+p_{z}\right)}{(n-1) \rho_{z}}\left(\delta^{(n-1)}-1\right)\right\}\right]
$$




\section{Results}

The typical variation of specific entropy production $\Delta s$ with the radial distance $R$ ' in upward direction $\left(\theta=0^{0}\right)$ at the depth of explosion $Z_{d}=4 \mathrm{~km}$ is shown in figure 2 . The corresponding variation of shock velocity is shown in figure 3 .

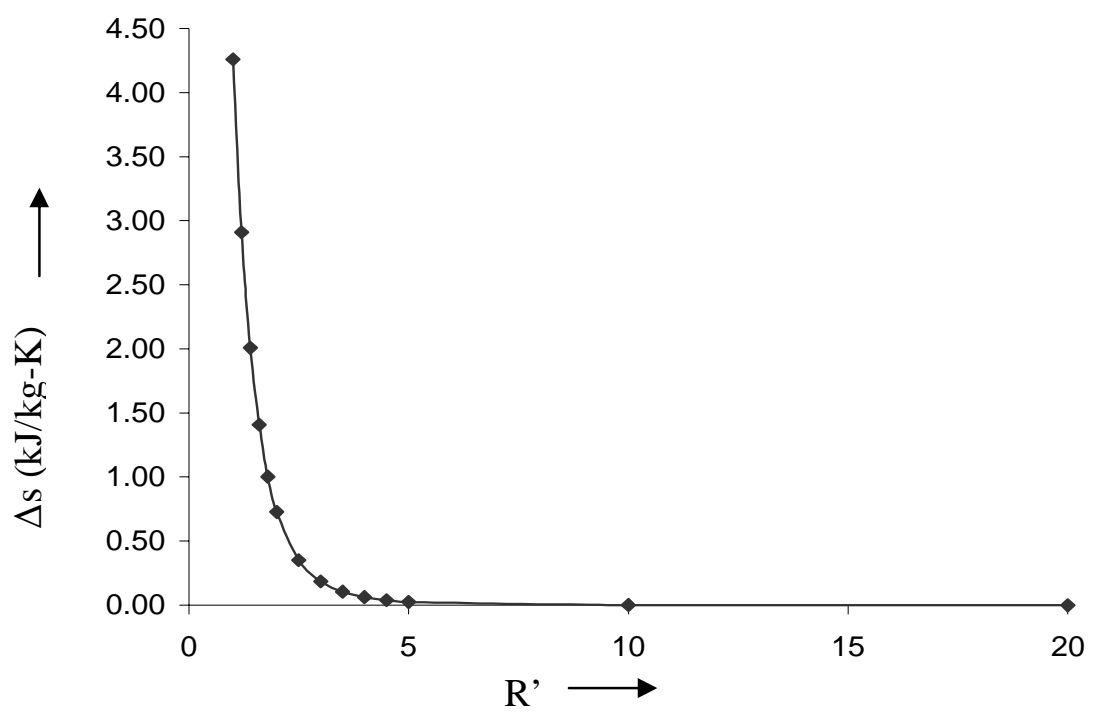

Figure 2-Entropy variation with radial distance

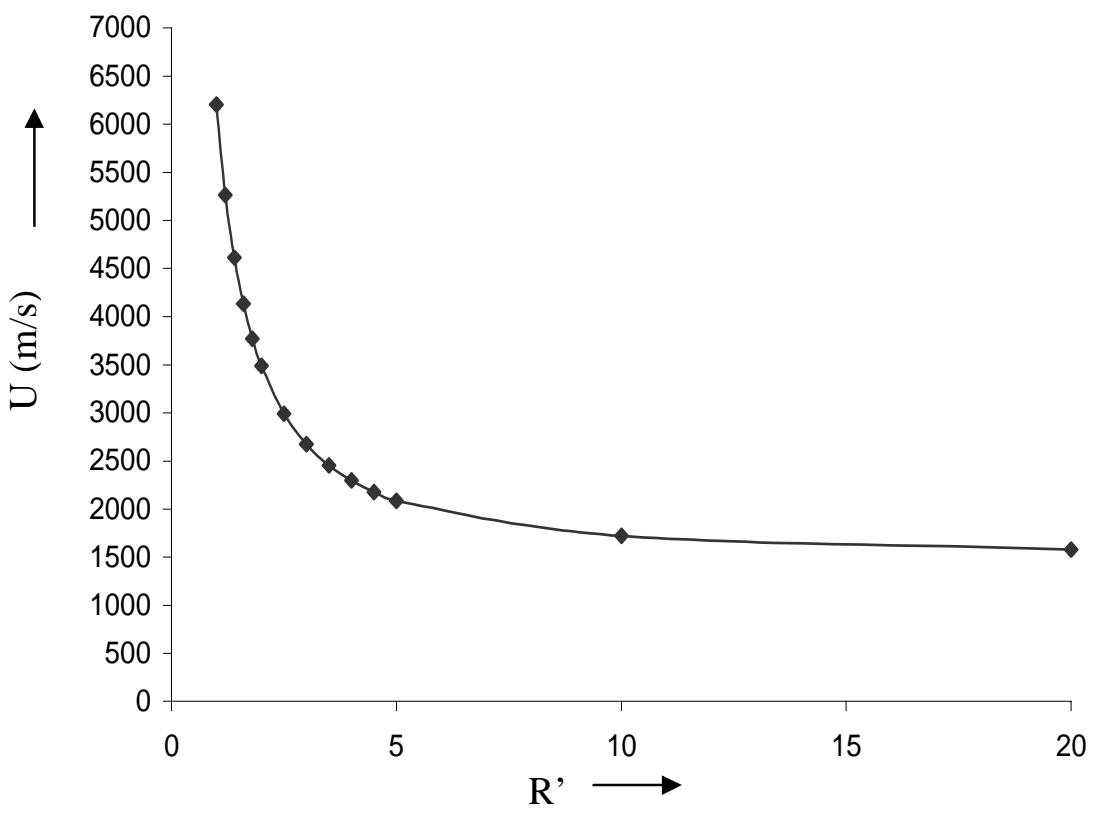

Figure 3- Shock velocity variation with radial distance 
Entropy variation also depends on the depth of explosion. Figure 4 shows the variation in entropy production with radial distance at different depths of explosions $\left(Z_{d}\right)$, as the shock moves upwards $\left(\theta=0^{0}\right)$.

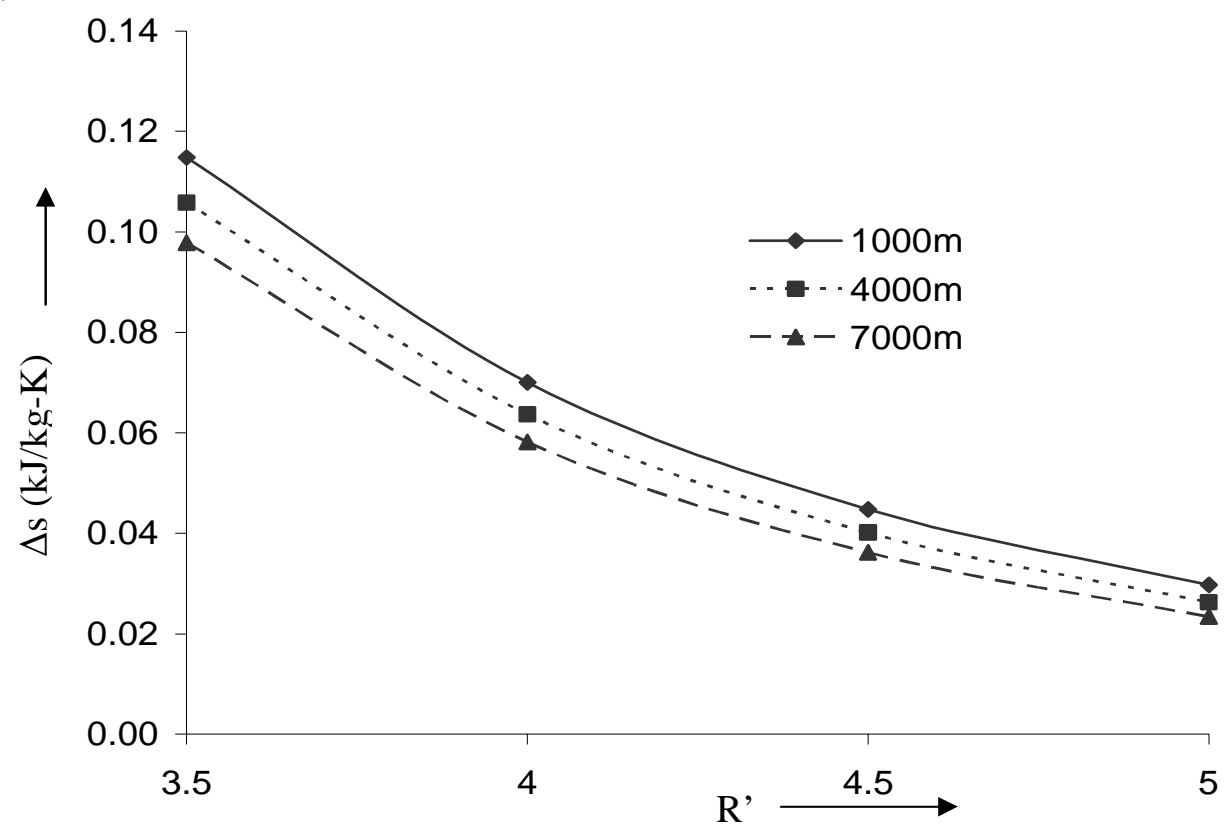

Figure 4- Effect of depth of explosion on entropy variation

Variation of entropy production with radial distance in different directions at the depth of explosion $Z_{d}=7000 \mathrm{~m}$ is shown in figure 5 .

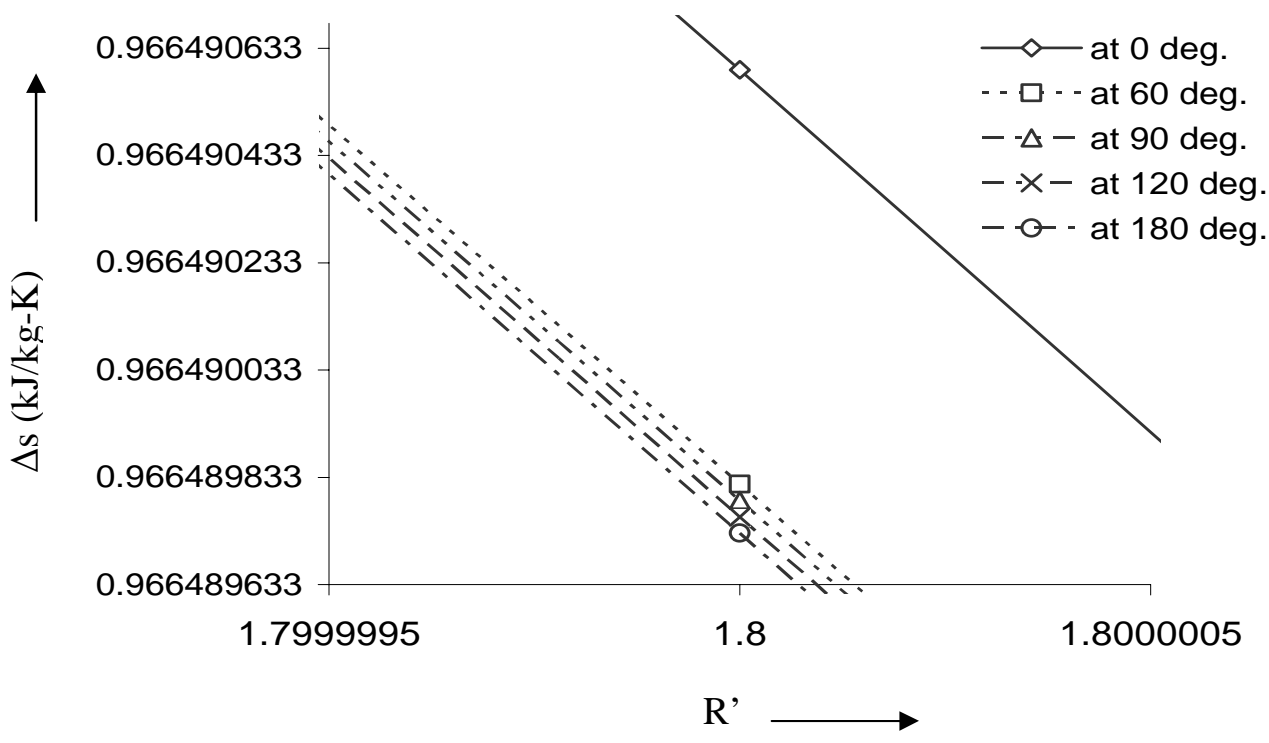

Figure 5- Typical variation of entropy with radial distance in different directions 
Figure 6 shows the variation of entropy production with the direction, at a fixed radial distance $\left(R^{\prime}=3.0\right)$, at a depth of explosion of $7000 \mathrm{~m}$. In it, $\Delta$ s axis is plotted at $\theta=90^{\circ}$, because for $\theta=90^{\circ}, z=Z_{d}$ i.e. shock moves horizontally. So the directions $<90^{\circ}$ signifies the upward motion of the shock, whereas the directions $>90^{\circ}$ signifies the downward motion of the shock.

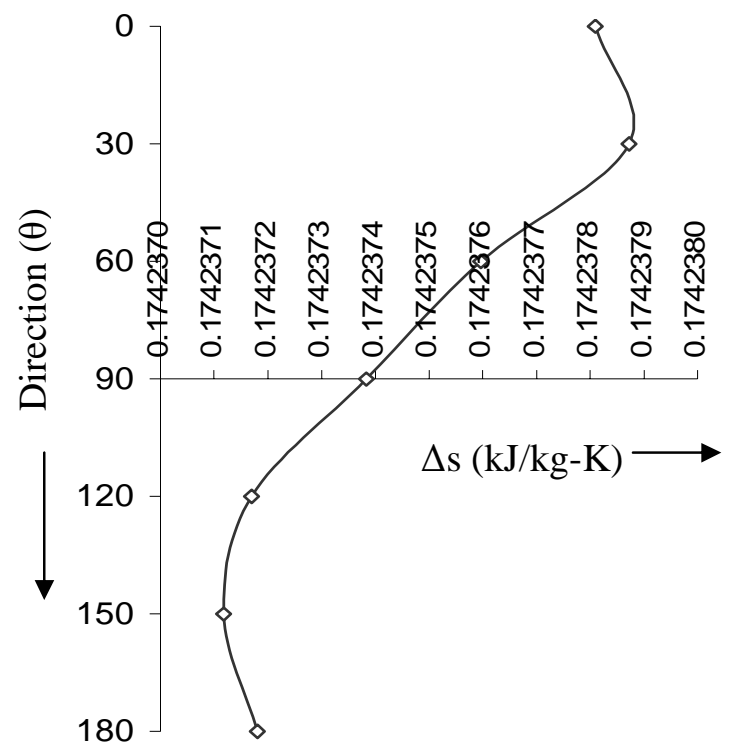

Figure 6- Variation in entropy production with direction (at $\mathrm{R}^{\prime}=3.0$ )

Shock velocity variation (Corresponding to figure 6) is shown in figure 7.

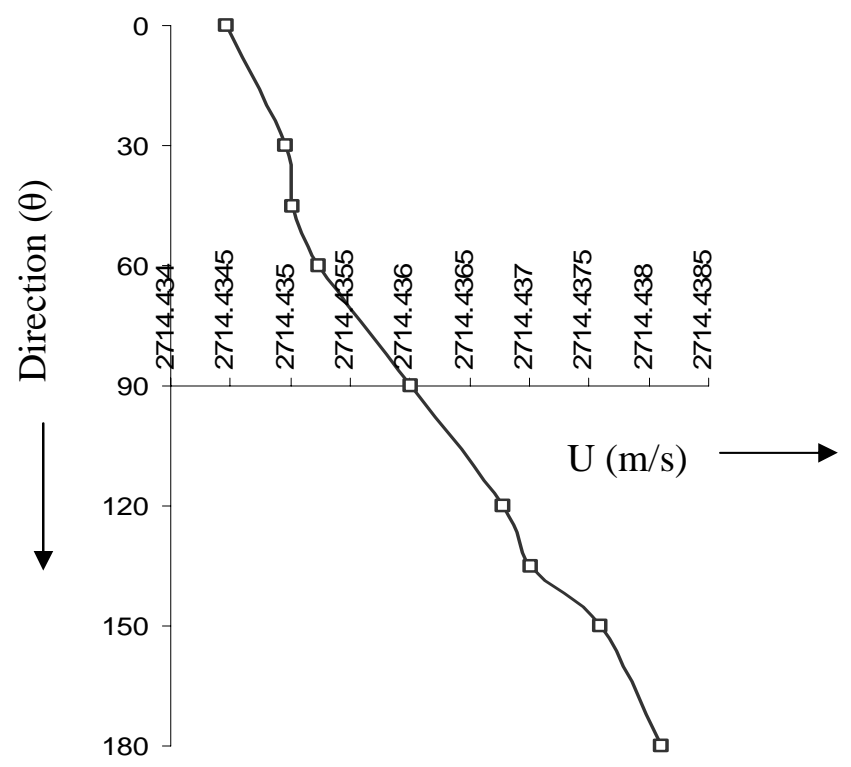

Figure 7- Variation in shock velocity with direction (at $\mathrm{R}^{\prime}=3.0$ )

Radial plots in figure 8 , shows the shock velocity and entropy profiles at different radial positions $\left(R^{\prime}=1.4,3.0\right.$ and 5.0) and at $\mathrm{Z}_{\mathrm{d}}=7000 \mathrm{~m}$. Radial axes show the different directions and the shaded area gives the magnitude of $\Delta s$ (and $\mathrm{U}$ ) in corresponding profiles. 

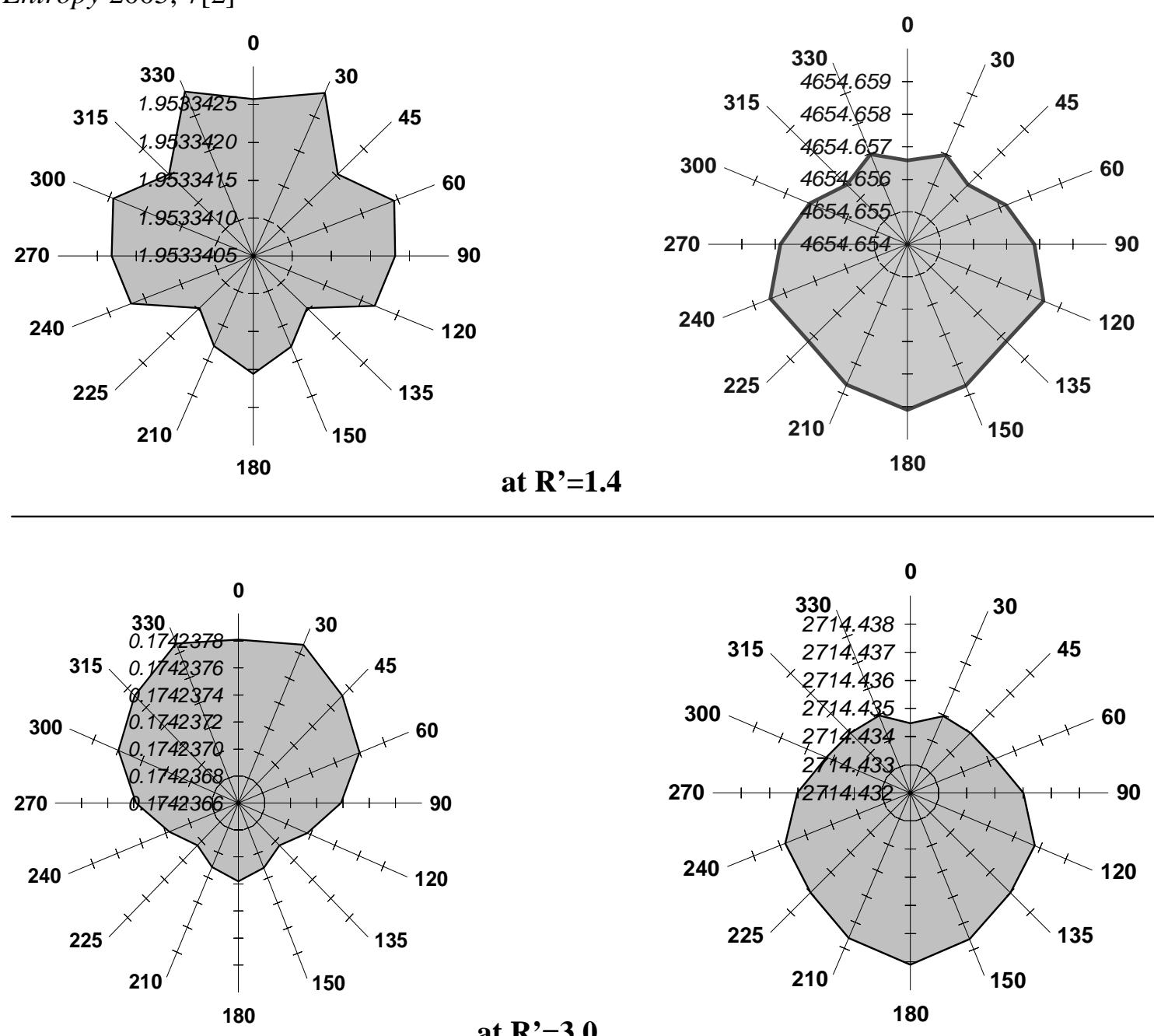

at $\mathbf{R}^{\prime}=3.0$
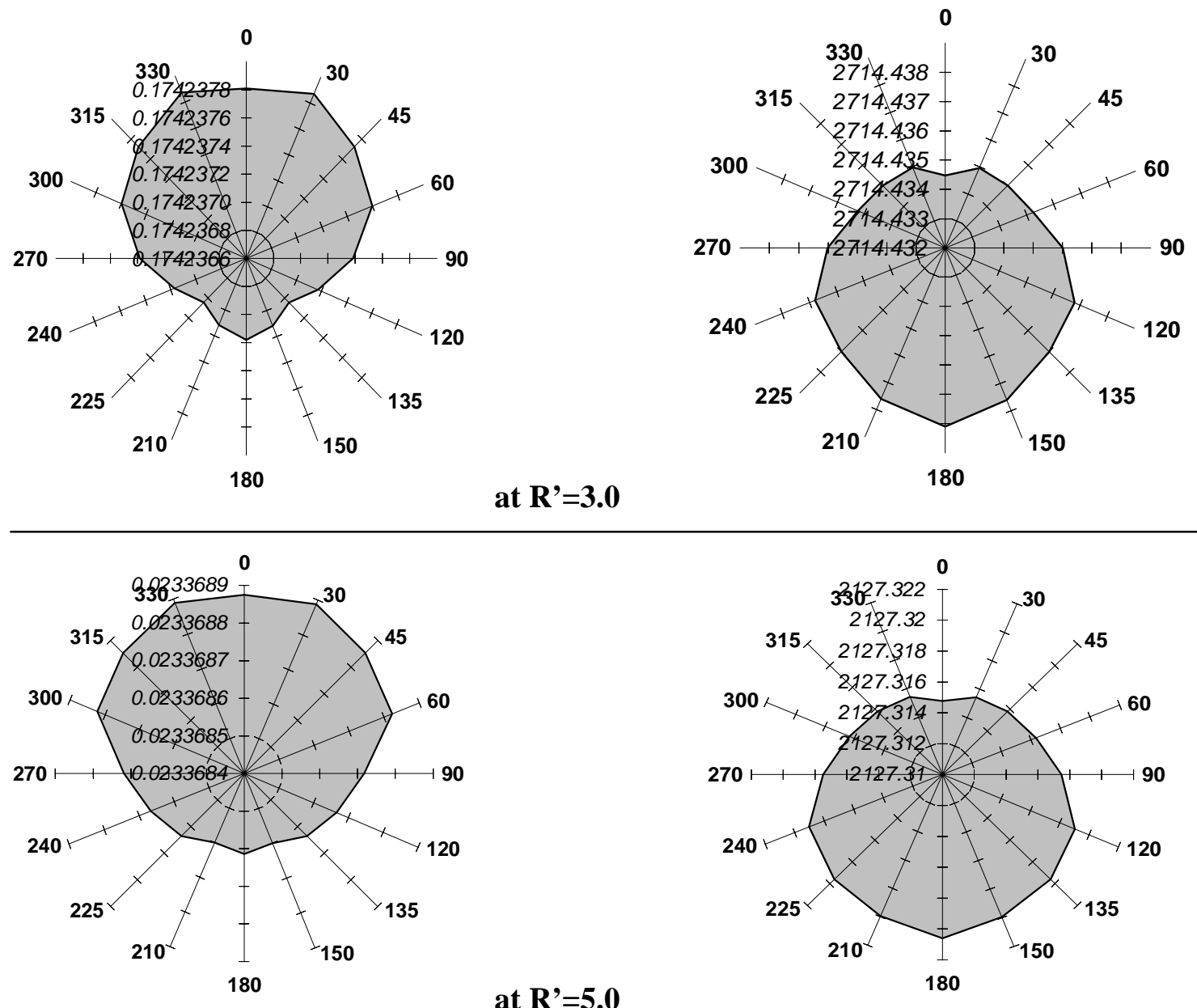

at $R^{\prime}=\mathbf{5 . 0}$
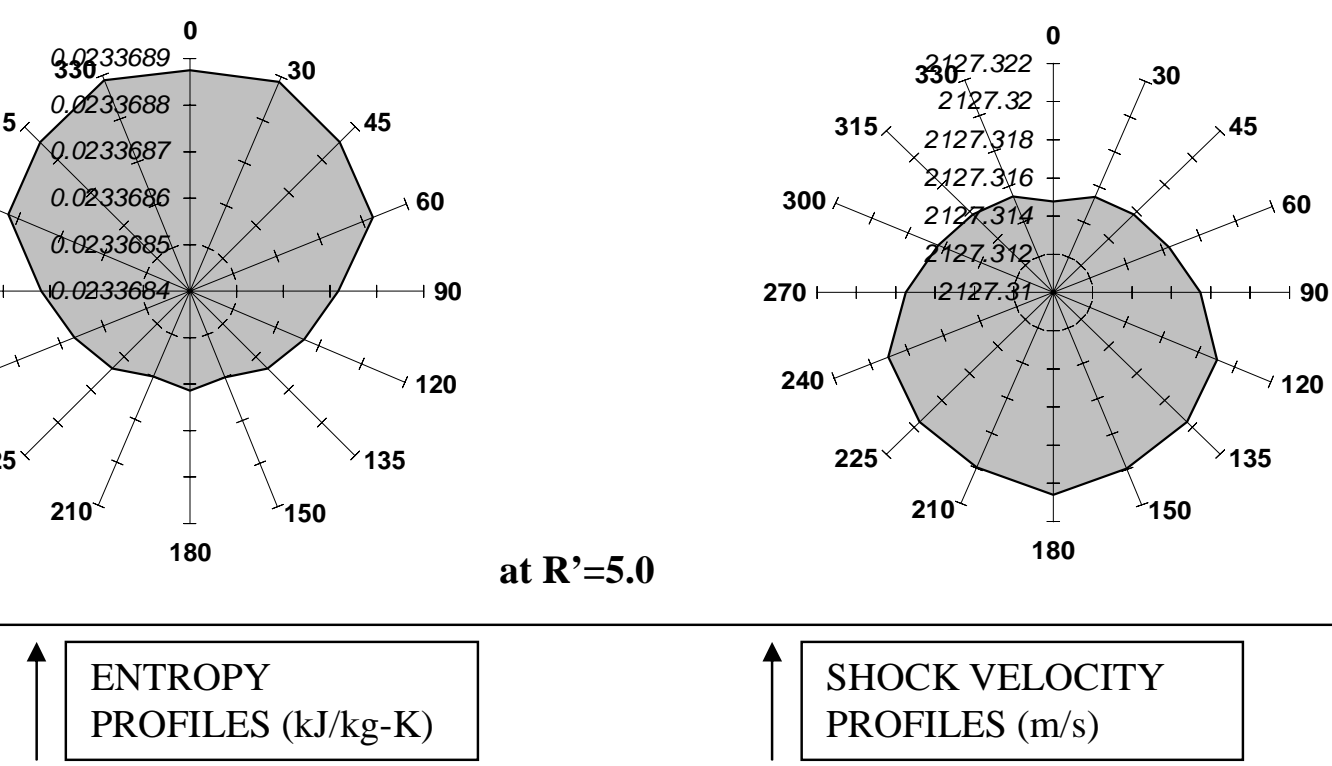

SHOCK VELOCITY PROFILES $(\mathrm{m} / \mathrm{s})$

Figure 8- Entropy profiles and corresponding shock velocity profiles at different radial Positions, as the shock moves away from the point of explosion 


\section{Analysis of results}

\section{Characteristics curves for blast waves:}

Figure 2 shows that as the shock moves away from the point of explosion, the entropy production is large near the point of explosion and it decreases very rapidly towards negligible value. At a distance $\mathrm{R}^{\prime}=5.0$, it is almost negligible. This is in agreement with figure 3 (between shock velocity and radial distance $\left.R^{\prime}\right)$. As $R^{\prime}$ increases, $U$ decreases and approaches the value of local sound velocity $(=1580.28$ $\mathrm{m} / \mathrm{s}$ ). These curves presents the basic nature of blast waves.

Propagation of shock in any medium produces a disordered motion of medium particles, which depends on the strength of the shock. An increase in the disorder of a medium is described by the increase in entropy $[8,26]$.As the shock moves away from the point of explosion its velocity decreases producing less disorder (Entropy) than its previous position. In this manner, the blast waves produce a non-isentropic flow field behind it, in which entropy is maximum at the explosive boundary.

\section{Effect of depth of explosion:}

Figure 4 shows that Explosion occurred at larger depths, produces a smaller change in entropy of water, then the explosion of same energy, at smaller depths. The corresponding dependence of shock velocity on depth of explosion is given in table 1.

\begin{tabular}{|c|c|c|c|}
\hline \multirow{2}{*}{$R^{\prime}$} & \multicolumn{3}{|c|}{$\begin{array}{r}\text { U at different } Z_{d} \text { 's in the direction } \\
\theta=0^{0}\end{array}$} \\
\cline { 2 - 4 } & $7000 \mathrm{~m}$ & $4000 \mathrm{~m}$ & $1000 \mathrm{~m}$ \\
\hline 1 & 6247.47 & 6206.10 & 6162.46 \\
1.2 & 5307.25 & 5265.28 & 5221.10 \\
1.4 & 4654.66 & 4612.42 & 4568.04 \\
1.6 & 4177.20 & 4134.86 & 4090.44 \\
1.8 & 3813.98 & 3771.62 & 3727.24 \\
2 & 3529.23 & 3486.89 & 3442.58 \\
2.5 & 3032.16 & 2989.93 & 2945.85 \\
3 & 2714.43 & 2672.31 & 2628.45 \\
\hline
\end{tabular}

Table 1- Shock velocity variation with depth of explosion

Table 1 show that shock moves with a larger velocity at larger depths. An increment of 1000m of depth produces a decrement in $\Delta s$ of the order of $10^{-3} \mathrm{~kJ} / \mathrm{kg}-\mathrm{K}$ and a large increment in $U$ of the order of $14 \mathrm{~m} / \mathrm{s}$.

This behavior can be explained well on the basis of static pressure $p_{z}$. As the depth increases, static pressure increases sharply. The large static pressure causes the shock front to produce lesser disordered motion of water molecules. In other words, entropy production decreases as the depth of explosion increases.

\section{Directional dependence of entropy production:}

Figure 5 shows that, in general when the shock moves upward $\left(\theta<90^{\circ}\right)$, entropy production is larger than the entropy production in downward direction $\left(\theta>90^{\circ}\right)$.

Figure 6 presents a relative difference of $\Delta s$, in different directions at a particular position of the shock front. This change in entropy is of the order $10^{-7} \mathrm{~kJ} / \mathrm{kg}-\mathrm{K}$. Figure 7 shows a relative difference of $U$ in 
different directions at a particular position of the shock front. This difference is of the order $10^{-4} \mathrm{~m} / \mathrm{s}$. These plots can be explained on the same ground as figure 4 .

In figure 8 , as $R$ ' increases, shock velocity $U$ decays and correspondingly $\Delta S$ also decrease. But in general, decay of shock in upward direction $\left(\theta<90^{\circ}\right)$ is faster than its decay in downward direction $\left(\theta>90^{\circ}\right)$. Whereas entropy production in upward directions is higher than downward directions of shock motion. It can also be seen that initially the profiles are not smooth. Velocity profiles shows fluctuations along $30^{\circ}$ and $45^{\circ}$. However entropy profile shows fluctuations along $30^{\circ}, 45^{\circ}$ and $135^{\circ}$ directions. As the radial distance increases, these fluctuation decreases and profiles shows smoothness in all directions.

\section{Conclusion}

The results of this paper show that the entropy production in seawater depends on the direction of shock propagation as well as on the depth of explosion. The results also shows that as the shock moves away from the point of explosion, the entropy production is larger near the point of explosion and it decreases very rapidly towards negligible value. At a distance of 5 radii of charge, entropy production is almost negligible.

The results obtained here are agreed with those given by Kirwood and Montroll [6]. However the estimation given in [6] was only for pure water at $20^{\circ} \mathrm{C}$, up to a pressure of $50 \mathrm{~kb}$. The effect of the depth of explosion and directional dependence was not considered in their estimation.

For the sake of simplicity, effect of the rotation of the earth is not considered here. Rotation of the earth produces centrifugal and Coriolis forces. Effect of centrifugal force is very small in comparison to gravitational force for the case of earth. Whereas horizontal component of Coriolis force produces ocean currents. We expect to include it in our next paper. Presence of the gas bubble at the point of explosion is also not considered. This gas bubble certainly affects the shock motion.

Shock propagation is an irreversible process with non-equilibrium states above and behind it. However from entropy point of view, a Local Thermo dynamical Equilibrium (LTE) is considered across the shock. The increase in entropy across the shock is an essential part of shock process and it is also a necessary condition for the stability of the shock [10]. The results of this paper also prove that irreversible process taking place inside a thermodynamic system always lowers the value of entropy production per unit time [15].

The most important aspect of the study of entropy production in seawater lies in the fact that, it presents the behavior of the medium particle (seawater) for the shock waves. Generation of entropy in this process causes the conversion of explosion energy into unavailable form. So less energy is available for shock propagation in the direction in which change in entropy is larger. In other words, this analysis provides the nonuniform distribution of explosion energy, available for shock propagation in different directions and at different depths.

These results are important in the modeling of Tsunami waves, caused by shock waves due to submarine earthquakes, landslides or volcanic eruptions. In this paper we have studied only a very small amount of explosive which liberates a small amount of explosion energy. However, a very large amount of energy is released in seismic and volcanic activities. In such cases, the effect of these results will be of considerable amount. Predictions and analysis of Tsunami waves can be improved using these results, which needs a further study in this field. 


\section{Data used:}

1. $\mathrm{R}_{\mathrm{e}}=6371230 \mathrm{~m}$

2. $\mathrm{g}_{\mathrm{s}}=9.81 \mathrm{~m} / \mathrm{s}^{2}$

3. $\mathrm{B}(s)=2.94 \mathrm{~kb}[4,11]$

4. $\mathrm{n}=7.25[4,11]$

5. $\mathrm{p}_{1}=1 \mathrm{~b}$

6. $\rho_{1}=1027 \mathrm{~kg} / \mathrm{m}^{3}[23]$

7. $T_{z}$ (average temperature in deep sea) $=276 \mathrm{~K} \mathrm{[23]}$

8. $\mathrm{c}_{\mathrm{p}}=4.186 \mathrm{~kJ} / \mathrm{kg}-\mathrm{K}$

9. Detonation data for the explosive RDX/TNT (60:40), [18]:-

- $R_{0}=0.0375 \mathrm{~m}$

- $\rho_{D}=1680 \mathrm{~kg} / \mathrm{m}^{3}$

- $p_{D}=255.528 \mathrm{~kb}$
- Mass of the charge $=0.365 \mathrm{~kg}$

- $U_{D}=7800 \mathrm{~m} / \mathrm{s}$

- $T^{\prime}=2252.11 \mathrm{~kJ}$

\section{References}

1. Berger, S.A.; Holt, M. Implosive phase of spherical explosion in sea water. Phy. Fluids 1962, 5, no.4, 426-431.

2. Bhatnagar, P.L.; Sachdev, P.L.; Prasad, P. Spherical piston problem in water. J. Fluid Mech. 1969, 39, 587-600.

3. Bhutani, O.P. Propagation and attenuation of a cylindrical blast wave in magnetogasdynamics. J. Math. Anal. Applic.1966, 13, 565-576.

4. Brand, R.S. Development in mechanics; Ostrach, S. and Scanlin, R.H.; 1965; vol.2, part1, p 507.

5. Buchanan, J.S.; James, H.J. Measurement of high intensity stress pulses. British J. Appl. Phys. 1959, 10, 290-295.

6. Cole, R.H.; Under water explosions; Dover, 1948; p 40.

7. Courant, R.; Friedrichs, K.O. Supersonic flow and shock waves; Interscience; 1948;p132.

8. Dincer, I.; Cengel, Y.A. Energy, Entropy and Exergy concepts and their role in thermal engineering; Entropy 2001; 3, 116-149.

9. Hartree, D.R. Numerical analysis; Oxford university press: London, 1962, 2 nd ed..

10. Henderson, L.F. On the thermodynamic stability of steady state adiabatic systems. J. Fluid Mech. 1988, 189, 509-529.

11. Hunter, C. On the collapse of an empty cavity in water. J. Fluid Mech. 1960, 8, 241-263.

12. Kirwood,J.G.; Bethe,H.A. Basic propagation theory. OSRD 588, 1942.

13. Kirwood,J.G.; Brinkley, S.R., Jr. Theory of the propagation of shock waves from explosive sources in air and water. OSRD 4814, 1945.

14. Penny, W.G.; Dasgupta, H.K. Underwater explosion research. ONRB report RC-333, 1942.

15. Prigogine, I. Introduction to thermodynamics of irreversible process; Interscience Pub., 1955; 2 nd ed., p 81.

16. Ranga Rav, M.P. \& Ramana, B.V. Self similar flow with increasing energy, forth piston driven flow of water. Int. J. Engng. Sci. 1973, 11, 1317-1327.

17. Resnick, R.; Halliday, D.; Walker J. Fundamentals of Physics; Willey eastern: New Delhi, 2004, p301.

18. Singh, V.P. Modification of energy hypothesis for the case of explosive charge. Ind. J. Pure Appl. Math. 1976, 7 no.2, 147-150.

19. Singh, V.P. Spherical shock wave in water. Ind. J. Phys. 1972, 46, 547-555.

20. Singh, V.P. On underwater explosions- A comparative study. Def. Sci. J. 1982, 32, 327-332.

21. Singh, V.P.; Bola, M.S. A note on explosive shock in homogeneous water. Ind. J. Pure Appl. Math. 1976, 7 no. 12, 1405-1410

22. Singh, V.P.; Madan, A.K.; Suneja, S.R.; Lal, C. Propagation of spherical shock wave in water. Proc. Ind. Acad. Sci.,(Engg. Sci.) 1980, 3, 169-175.

23. The times atlas of the oceans, Van nostrand reinhold co.: New York, 1983. 
24. Thomas, T.Y. On the propagation and decay of spherical blast waves. J. Math. Mech. 1957, 6, 607-620.

25. Vishvakarma,J.P.; Nagar, K.S.; Mishra, R.B. On the propagation of shock wave produced by explosion of a spherical charge in deep sea. Def. Sci. J. 1988, 38, no.1, 69-76.

26. Zemansky, M.W.; Dittman, R.H. Heat and thermodynamics; Mc Graw Hill.: NewYork,1997; pp 52-53, 267, 215.

(C) 2005 by MDPI (http://www.mdpi.org). Reproduction for noncommercial purposes permitted. 